\title{
Correlations of iodide ions with vascular endothelial growth factor and its receptors during the proliferation of vascular endothelial cells
}

\author{
F. Teng, M.H. Zu and Q.J. Hua \\ Department of Interventional Radiography, \\ The Affiliated Hospital of XuZhou Medical College, Xuzhou, China \\ Corresponding author: M.H. Zu \\ E-mail: maohengzu@163.com
}

Genet. Mol. Res. 13 (3): 6439-6447 (2014)

Received June 11, 2013

Accepted October 22, 2013

Published August 25, 2014

DOI http://dx.doi.org/10.4238/2014.August.25.7

\begin{abstract}
The aim of this study was to explore the correlations of iodide ions with vascular endothelial growth factor (VEGF) and its receptors during the proliferation of vascular endothelial cells (VECs). The proliferation rates of VECs in the presence of iodide ions and VEGF inhibitor were determined using the CCK-8 method. The effect of iodide ions on the phosphorylation of vascular endothelial growth factor receptor 2 (VEGFR-2) was observed using Western blot analysis. In the presence of $300 \mu \mathrm{g} / \mathrm{L}$ iodide ions, the application of VEGF inhibitor did not inhibit VEC proliferation $(\mathrm{P}<0.05)$. At a certain concentration, iodide ions upregulated the phosphorylation level of VEGFR-2 at the Tyr1214 site $(\mathrm{P}<0.05)$. Iodide ions did not influence the phosphorylation of VEGFR-2 at the Tyr1175 and Tyr951 sites. At an appropriate concentration, iodide ions serve as an independent VEC proliferation-promoting factor. They mediate VEC migration by stimulating the upregulation of the phosphorylation level of VEGFR-2 (Tyr1214) and do not influence VEGFR-2
\end{abstract}


phosphorylation at Tyr1175. Therefore, their VEC proliferationpromoting effect is independent of membrane receptors.

Key words: Budd-Chiari syndrome; Vascular endothelial cell; Iodide ion; Vascular endothelial growth factor (VEGF); VEGF receptor 2

\section{INTRODUCTION}

Budd-Chiari syndrome (BCS) is caused by hepatic venous obstruction and/or inferior vena cava obstructive lesions above the junction, and belongs to a type of posthepatic portal hypertension characterized by portal hypertension and/or inferior vena cava hypertension (MacNicholas et al., 2012). In China, BCS is the most common type of membranous obstruction of the inferior vena cava (MOVC) whose development is characterized by septum formation above the junction of the inferior vena cava and hepatic veins with vascular endothelial tissues on both the upper and lower surfaces of the septum. However, how septa with varying thickness can develop, given the thickness of the inferior vena cava and constant blood flow, has long puzzled clinicians and been a hotspot and challenge of basic BCS research. According to an epidemiological study conducted in China, patients with MOVC type BCS are primarily found living in places where the iodine content in water exceeds the normal standard (Guo et al., 2005). Another study demonstrated that BCS patients have higher than average iodine concentrations in their blood and urine (Jin et al., 2005). Iodide ions induce the expression of vascular endothelial growth factor (VEGF) and cellular inflammatory factors (Ohtani et al., 2007). These findings indicate that the development of MOVC type BCS is closely correlated with the dysbolism of the microelement iodine.

In this study, we cultured vascular endothelial cells (VECs) in vitro and then explored the action mechanism of iodide ions underlying their proliferation. The aim of this study was to provide a new perspective of the cause of inferior vena cava membranous formation in patients with MOVC type BCS.

\section{MATERIAL AND METHODS}

\section{Cell culture}

EA. Hy926 human umbilical vein endothelial fusion cells (Catalogue no.: GNHu39) were purchased from the cell resource center of the Shanghai Institute for Biological Sciences, Chinese Academy of Sciences. The cells were cultured in high glucose Dulbecco's modified Eagle's medium (DMEM) (GIBCO; USA) in a Corning culture dish (USA) to which $10 \%$ fetal calf serum (GIBCO) was added. The cells were allowed to stand in $5 \% \mathrm{CO}_{2}$ at $37^{\circ} \mathrm{C}$ until adherence was reached for passage. This study was conducted in accordance with the Declaration of Helsinki and with approval from the Ethics Committee of XuZhou Medical College. Written informed consent was obtained from all participants.

\section{CCK-8 for cell proliferation detection}

VECs in logarithmic growth phase were digested for cell suspension preparation. 
The cells were counted using a cell counting chamber for cell density determination. The density was diluted to $5 \times 10^{4} / \mathrm{mL}$. DMH4 (VEGF inhibitor; Sigma; USA) solution at 4.8 $\mathrm{mg} / \mathrm{mL}$ was prepared with dimethyl sulfoxide (DMSO; Sigma) as the solvent according to the requirement. Working solution with a $7.2 \mu \mathrm{g} / \mathrm{mL}$ iodide ion concentration was prepared using analytically pure potassium iodide (Sinopharm; China). The cell suspension was inoculated onto a 96-well plate with around 5000 cells in each well. The cells were divided into blank control (100 $\mu \mathrm{L}$ cell suspension and $20 \mu \mathrm{L}$ DMEM were added to each well), solvent ( $100 \mu \mathrm{L}$ cell suspension, $19 \mu \mathrm{L}$ DMEM, and $1 \mu \mathrm{L}$ DMSO), KI (100 $\mu \mathrm{L}$ cell suspension, $15 \mu \mathrm{L}$ DMEM, and $5 \mu \mathrm{L} \mathrm{KI})$, DMH4 $+\mathrm{KI}(100 \mu \mathrm{L}$ cell suspension, $10 \mu \mathrm{L}$ DMEM, $5 \mu \mathrm{L}$ DMH4, and $5 \mu \mathrm{L} \mathrm{KI})$, and DMH4 (100 $\mu \mathrm{L}$ cell suspension, $15 \mu \mathrm{L}$ DMEM, and $5 \mu \mathrm{L}$ DMH4) groups, where the KI and DMH4 + KI groups had $300 \mu \mathrm{g} / \mathrm{L}$ final iodide ion concentrations and the DMH4 + KI and DMH4 groups had $5 \mu \mathrm{M}$ final DMH4 concentrations. Eight replicate pores were set for each group. All groups were allowed to stand in $5 \% \mathrm{CO}_{2}$ at $37^{\circ} \mathrm{C}$ for $12 \mathrm{~h}$. The media were replaced and $10 \mu \mathrm{L}$ CCK- 8 working solution was added to each pore for $30 \mathrm{~min}$ of incubation. A DMEM + CCK-8 pore was set to zero as the background. Optical density (OD) was read at a wavelength of $450 \mathrm{~nm}$. The maximum and minimum OD values were excluded from each group. The relative growth rate of each group was calculated as the ratio between the OD values of the experimental and blank control groups. The experiment was repeated 3 times.

\section{Western blot analysis of vascular endothelial growth factor receptor 2 (VEGFR-2) phosphorylation}

VECs in logarithmic phase were passaged and then cultured routinely until $70 \%$ fusion was reached. Afterward, they were cultured with serum-free DMEM for $24 \mathrm{~h}$. The cells were divided into blank control and iodide ion groups, according to the iodide ion concentrations added. The control group was directly cultured in DMEM medium. For the iodide ion groups, potassium iodide was added to DMEM to obtain 100, 300, 500, and $1000 \mu \mathrm{g} / \mathrm{L}$ final iodide ion concentrations, respectively. The cells were cultured for 1 $\mathrm{h}$ and then washed with phosphate-buffered saline (PBS) 3 times. Proteinase and $60 \mu \mathrm{L}$ phosphatase inhibitor-containing radio-immunoprecipitation assay lysis buffer was added to each culture dish for total protein extraction. After concentration determination using the bicinchoninic acid method, total protein was subpackaged. After sodium dodecyl sulfate (SDS) loading buffer was added, the fractioned samples were boiled at $100^{\circ} \mathrm{C}$ for 5 min. Then, they were subjected to application, electrophoresis, transmembrane, and immune reactions. The primary antibodies were anti-VEGFR-2 antibody (Abcam; UK), antiphospho-VEGFR-2 (Tyr1175) antibody (Cell Signal Technology; USA), anti-VEGFR-2 (phospho Y1214) antibody (Abcam), anti-phospho-VEGFR-2 (Tyr951) antibody (Cell Signal Technology), and anti- $\beta$-actin antibody (Abcam), respectively. The secondary antibody was alkaline phosphatase anti-rabbit IgG (Vector Laboratories, USA). The samples were stained using 5-bromo-4-chloro-3-indolyl-phosphate/nitrotetrazolium blue chloride, and $\beta$-actin was taken as internal reference. Specific bands were semiquantitatively analyzed using the IPP6.0 image analysis software. The expressions of VEGFR-2 and phosphoVEGFR-2 proteins were determined by the ratio of VEGFR-2 and phospho-VEGFR-2 to $\beta$-actin, respectively. The experiment was repeated 3 times. 


\section{Statistical analysis}

All data are reported as means \pm standard error. They were statistically analyzed by using the SPSS16.0 software. One-factor analysis of variance (ANOVA) was performed for comparisons among groups, and least significant difference (LSD) was used for comparisons between experimental groups and the control group. $\mathrm{P}<0.05$ was considered to be statistically significant.

\section{RESULTS}

\section{Effect of iodide ions + DMH4 on VEC proliferation}

No significant difference was observed between the VEC proliferation rates of the control and solvent groups $(\mathrm{P}>0.05)$. This suggested that DMSO had no influence on the experiment. Compared with the control group, the DMH4 group showed a significantly decreased VEC proliferation rate $(\mathrm{P}<0.05)$. This finding suggested that DMH4, as a VEGF inhibitor, inhibits VEC proliferation by inhibiting the activity of VEGF. By contrast, the KI group showed a significantly increased proliferation rate $(\mathrm{P}<0.05)$. This finding suggested that $300 \mu \mathrm{g} / \mathrm{L}$ iodide ions stimulate cell proliferation. Similarly, the DMHR + KI group displayed a significant increase in the cell proliferation rate $(\mathrm{P}<0.05)$, and this increase was comparable to that in the KI group $(\mathrm{P}>0.05)$. This finding suggested the cell proliferation rate still increased in the presence of $300 \mu \mathrm{g} / \mathrm{L}$ iodide ions even after the activity of VEGF was inhibited. These results demonstrated that $300 \mu \mathrm{g} / \mathrm{L}$ iodide ions stimulate VEC proliferation and that the inhibition of VEGF activity cannot prevent VEC proliferation in the presence of $300 \mu \mathrm{g} / \mathrm{L}$ iodide ions. The results are summarized in Table 1 .

\begin{tabular}{|c|c|}
\hline Group & Relative proliferation rate \\
\hline Blank control & $1.00 \pm 0.00$ \\
\hline Solvent & $0.94 \pm 0.06$ \\
\hline KI & $1.52 \pm 0.12$ \\
\hline DMH4 + KI & $1.50 \pm 0.13$ \\
\hline DMH4 & $0.57 \pm 0.07$ \\
\hline
\end{tabular}

Data are reported as means \pm standard error.

\section{Effects of iodide ions on VEGFR-2 expression and phosphorylation at the Tyr951, Tyr1175, and Tyr1214 sites}

The results of the Western blot analysis are shown in Table 2 and Figure 1.

None of the iodide ion groups displayed a significant difference in VEGFR-2 expression compared with the blank control group $(\mathrm{P}>0.05)$. No significant differences were observed among the iodide ion groups $(\mathrm{P}>0.05)$. These findings indicate that iodide ions have no influence on VEGFR-2 expression.

Compared with the control group, all the iodide ion groups displayed a decrease in the phosphorylation level of VEGFR-2 at Tyr1214 $(\mathrm{P}<0.05)$. Compared with the $100 \mu \mathrm{g} / \mathrm{L}$ 
group, the 300,500 , and $1000 \mu \mathrm{g} / \mathrm{L}$ groups showed increases in phosphorylation $(\mathrm{P}<0.05)$, whereas no noticeable differences were observed among them $(\mathrm{P}>0.05)$. These findings indicate that iodide ions upregulate the phosphorylation level of VEGFR-2 (Tyr1214) to varying degrees.

Table 2. Effects of iodide ions on the relative expression of VEGFR-2 total protein and phosphorylated proteins at the Tyr 1214 site $(\mathrm{N}=3)$.

\begin{tabular}{|c|c|c|}
\hline Group & Relative expression of VEGFR-2 total protein & Relative expression of phosphorylated proteins at the Tyr1214 site \\
\hline Blank control & $3.85 \pm 1.41$ & $0.04 \pm 0.00$ \\
\hline $\mathrm{I}^{-} 100 \mu \mathrm{g} / \mathrm{L}$ & $3.99 \pm 1.73$ & $0.10 \pm 0.01$ \\
\hline $\mathrm{I}-300 \mu \mathrm{g} / \mathrm{L}$ & $3.38 \pm 0.80$ & $0.18 \pm 0.01$ \\
\hline $\mathrm{I}-500 \mu \mathrm{g} / \mathrm{L}$ & $3.50 \pm 1.45$ & $0.18 \pm 0.01$ \\
\hline $\mathrm{I}-1000 \mu \mathrm{g} / \mathrm{L}$ & $3.51 \pm 0.71$ & $0.21 \pm 0.04$ \\
\hline
\end{tabular}

Data are reported as means \pm standard error.

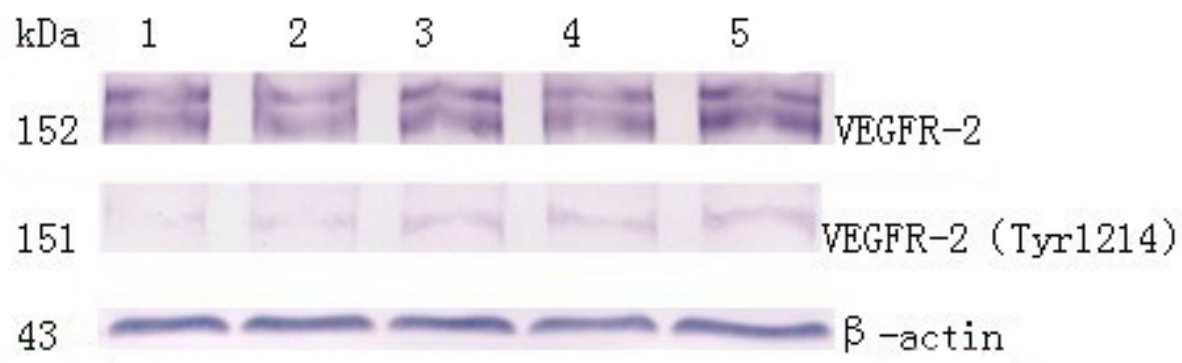

Figure 1. Effects of iodide ions on VEGFR-2 expression and the phosphorylation level at the Tyr1214 site. Lane 1 $=$ the blank control group; lane 2 = the $100 \mu \mathrm{g} / \mathrm{L}$ group; lane $3=$ the $300 \mu \mathrm{g} / \mathrm{L}$ group; lane $4=$ the $500 \mu \mathrm{g} / \mathrm{L}$ group; lane $5=$ the $1000 \mu \mathrm{g} / \mathrm{L}$ group.

For the other 2 phosphorylation sites, Tyr951 and Tyr1175, the Western blot analysis did not show phosphorylated blots in the control group or the induction of phosphorylation changes by iodide ions.

\section{DISCUSSION}

BCS is caused by hepatic venous obstruction and/or inferior vena cava obstructive lesions above the junction, and belongs to a type of post-hepatic portal hypertension characterized by portal hypertension and/or inferior vena cava hypertension (MacNicholas et al., 2012). In China, MOVC is the most common type of BCS whose development is characterized by membranous formation above the junction of the inferior vena cava and hepatic veins with vascular endothelial tissues on both surfaces of the membrane. In recent years, etiological studies of BCS have focused on genetic thrombosis caused by the mutation of genes such as $61691 A$ (clotting factor V gene; the mutation is also known as the FVLeiden mutation) (Slusher, 2010), G20210A (prothrombin gene; FIIG20210A) (Wlazłowski et al., 2011), JAK2-V617F (Tondeur et al., 2010; Smira et al., 2010; Smalberg et al., 2011), and so on. These causes are closely related to hepatic vein thrombosis and hepatic venous occlusion 
(Pasmant et al., 2010). In addition, thrombophlebitis, erythrocytosis, multiple myeloma, and positive antiphospholipid antibody due to long-term oral administration of contraceptives are other causes of these medical conditions (Carvalho et al., 2011; Yadav et al., 2012). Although the aforementioned studies have identified BCS manifested by hepatic venous obstruction due to thrombosis, they cannot provide an explanation for that manifested by MOVC. Therefore, membranous formation has become a hotspot as well as a challenge of BCS research. Considering the fact that both surfaces of the membrane are composed of vascular endothelial tissues, it is reasonable to believe that the abnormal proliferation and migration of VECs are the key factors involved in membranous formation.

According to an epidemiological study conducted in China, the geographical distribution of MOVC type BCS reported basically matches the regional distribution of excessive iodine in water. Urine iodine determination shows that BCS patients have a higher level of urine iodine than healthy people. Presumably, BCS patients have taken in excessive iodine through drinking and eating. After reducing to iodide ions in the intestinal tract, iodine is absorbed promptly by the intestinal tract into blood plasma and then into the liver by means of the portal vein. There, part of the iodine is delivered to the inferior vena cava through the hepatic veins, whereas the other part is packed in bile by the liver and then delivered to the digestive tract, constituting hepatoenteral circulation. For this reason, the iodide ion concentration at the junction of the hepatic veins becomes the highest in the human circulation system. Meanwhile, blood containing a high concentration of iodide ions impacts the walls of the inferior vena cava causing repeated injury, repair, and hyperplasia of VECs, which ultimately lead to membrane-like tissue formation. Therefore, iodide ions may be an important factor of membranous formation in the inferior vena cava in BCS patients. The results of this study showed that iodide ions, at an appropriate concentration, can be considered as an independent factor of VEC proliferation and that they mediate VEC migration by stimulating the upregulation of the phosphorylation level of VEGFR-2 (Tyr1214), which are consistent to what was expected.

Iodide ions, at a certain concentration, can promote the proliferation of VECs (Wang et al., 2007). To determine whether there is a synergetic effect between iodide ions and VEGF, DMH4 and iodide ions at an appropriate concentration were concomitantly used to act on VECs in this study. The results showed that iodide ions promote the proliferation of VECs independently of the stimulation of VEGF to membrane receptors. They had such an effect even after the activity of VEGF was inhibited. At an appropriate concentration, iodide ions can be considered as a VEC proliferation promoting factor. Therefore, iodide ions presumably serve as extracellular stimuli, which cause changes in the extracellular environment, directly or indirectly leading to the phosphorylation of membrane receptors.

VEGFRs are important receptors of VECs and play critical regulatory roles in the proliferation and apoptosis of VECs. In the VEGFR family, tyrosine protein kinase receptors consist of 3 subtypes: VEGFR-1, VEGFR-2, and VEGFR-3. VEGFR-1 has low endothelial cell-regulating activity. VEGFR-2 is principally distributed in VECs and lymphatic endothelial cells and has high activity. VEGFR-3 is primarily expressed in lymphatic endothelial cells. The stimulatory effect of the first messenger on the proliferation and apoptosis of VECs and its regulatory effects on the migration and permeability of VECs are realized by binding and activating VEGFR-2 (Shibuya, 2006).

VEGFR-2 is a type III transmembrane protein kinase whose activation is achieved by means of phosphorylation. VEGFR-2 possesses several phosphorylation sites. The phosphory- 
lation at different sites induces the activation of different downstream signaling proteins, leading to corresponding physiological or pathological changes. Among different phosphorylation sites, the important ones are Tyr951, Tyr1175, and Tyr1214, which have different downstream pathways (Matsumoto and Mugishima, 2006). Tyr951 is mainly responsible for VEC migration. It is located in the kinase insert region and can bind with T cell-specific adapter protein after phosphorylation to form compounds causing cell migration. Tyr1175 is mainly responsible for VEC proliferation. It is located at the C end of VEGFR-2. After phosphorylation, it binds with PLC-y causing the phosphorylation of PLC-y. Then, PLC-y dehydrolyzes phosphatidylinositol 4,5-diphosphate to generate diacylglycerol (DAG). DAG is an activator of protein kinase $C$ that plays a critical role in the Raf-MEK-ERK sequential activation. Tyr1214 is also located at the $\mathrm{C}$ end of VEGFR-2. It contains a pY-D-N-T sequence anastomotic to the Src homology domain 2 (SH2) of growth factor receptor binding protein-2 (Grb2). After phosphorylation, it can bind with cell division cycle protein-42 (Cdc42) and p38 mitogen-activated protein kinases (p38MAPK) in succession to mediate intra-VEC actin remodeling, stress fiber formation, and VEC migration (Lamalice et al., 2004).

In this study, iodide ions and VECs were concomitantly cultured in vitro and the stimulatory effect of iodide ions on cell membranes was observed. The Western blot analysis did not show phosphorylated protein blots at the Tyr1175 site, which contradicted with what had been expected. As the Tyr1175 site and its downstream signaling pathway mediate VEC proliferation-promoting effect, it was expected that the VEC proliferation promoting effect of iodide ions would be realized by stimulating VEGFR-2 to induce phosphorylation at its Tyr1175 site. However, the results revealed that the VEC proliferation-promoting effect of iodide ions bears no relation to phosphorylation at the Tyr1175 site. Instead, they may influence other apoptosis-regulating proteins such as the Bcl-2 family of proteins (Durdu et al., 2012) and the Fas/FasL protein (Yamana et al., 2005), as well as their signal transduction pathways.

For the 2 phosphorylation sites regulating VEC migration, the Western blot analysis did not show phosphorylation blots at Tyr951, but it did at Tyr1214. Furthermore, the experimental groups displayed significant differences in the phosphorylation levels compared with the control group. These results indicate that iodide ions increase the phosphorylation level of VEGFR-2 (Tyr1214) and bind with the SH2 of Grb2 to activate the Cdc42/p38MAPK signal pathway, thereby causing intra-VEC actin remodeling, stress fiber formation, and VEC migration. This indication is in line with vascular lesions in BCS patients; the inferior vena cava walks longitudinally, whereas the inferior vena cava membrane extends perpendicularly to the vessel wall. The inferior vena cava membrane may originate at the cellular level; apart from VEC proliferation, there should also be VEC horizontal migration toward the lumen.

Septum tissue biopsy has shown that the surface of the septum is composed of VECs. Beneath the surface is fibrous connective tissue and then dense connective tissue and elastic fibers (the deep layer) (Wang et al., 2008). Based on the results obtained from the clinical biopsy and the in vitro culture experiment, the hepatic veins in patients with MOVC type BCS may have excessively large junction angles, which cause changes in the blood shear stress at the junction sites. Such changes lead to damage to the VECs present there. Changes in the blood biochemical environment, such as a high concentration of blood iodine, further aggravate VEC migration toward the vessel lumens. Furthermore, the exposure of the subendothelial connective layer (containing fibrocytes, elastic fibers, and so on) to blood can result in repair to the damage. Consequently, static fibrocytes turn to functionally active fibroblasts, which proliferate 
for repair, secrete collagen fibers, and ultimately lead to the formation of the connective layer of the septum. The connective tissue layer is another important component of the septum and fibroblasts play an important role during its formation. Iodide ions can also promote the formation of collagen fibers (Capriotti and Capriotti, 2012). However, the concrete mechanisms of iodide ions underlying fibroblast proliferation and collagen fiber formation remain to be explored.

In summary, based on the epidemiological characteristics of the high incidence rate of MOVC type BCS in China and taking VEGFR-2 phosphorylation as the breakthrough point, this study elucidated the possible pathogenesis of MOVC type BCS from the perspectives of the proliferation and migration of VECs, thus providing a new idea for the basic research of this type of BCS. Meanwhile, the clinical biopsy results also demonstrated the significance of this study for revealing the causes of BCS. In addition, hepatic venous BCS is more commonly seen in western countries, which is characterized by the extensive (or local) formation of hepatic venous thrombi, obstruction, and stenosis. As this type of BCS is primarily caused by thrombosis, studies in western countries have focused on the causes of hepatic venous thrombosis such as gene mutations at certain sites. However, considering the fact that there are also cases of MOVC type BCS characterized by septum formation in these countries, gene mutations cannot provide satisfactory explanations. Different types of BCS have different pathogeneses, and thus the selection of a breakthrough point should depend on the types of BCS considered. This study has a limitation: since mainly focusing on MOVC type BCS, these results cannot completely explain the pathogenesis of simple hepatic venous BCS characterized by hepatic venous thrombosis.

\section{REFERENCES}

Capriotti K and Capriotti JA (2012). Topical iodophor preparations: chemistry, microbiology, and clinical utility. Dermatol. Online J. 18: 1.

Carvalho D, Oikawa F, Matsuda NM and Yamada AT (2011). Budd-Chiari syndrome in association with Behçet's disease: review of the literature. São Paulo Med. J. 129: 107-109.

Durdu S, Deniz GC, Balci D, Zaim C, et al. (2012). Apoptotic vascular smooth muscle cell depletion via BCL2 family of proteins in human ascending aortic aneurysm and dissection. Cardiovasc. Ther. 30: 308-316.

Guo CH, Bian JC, Wang Y, Bao R, et al. (2005). Effects of multiple elements in drinking water on inferior vena cava membranous obstruction type of the Budd-Chiari syndrome in Heze area of Shandong Province. Chin. J. Endemiol. 24: 207-209.

Jin LM, Bian JC, Wang Y, Bao R, et al. (2005). Study on relation between the Budd-Chiari syndrome and urine iodine in Heze area of Shandong Province. Chin. J. Endemiol. 20: 238-240.

Lamalice L, Houle F, Jourdan G and Huot J (2004). Phosphorylation of tyrosine 1214 on VEGFR2 is required for VEGFinduced activation of Cdc42 upstream of SAPK2/p38. Oncogene 23: 434-445.

MacNicholas R, Olliff S, Elias E and Tripathi D (2012). An update on the diagnosis and management of Budd-Chiari syndrome. Expert. Rev. Gastroenterol. Hepatol. 6: 731-744.

Matsumoto T and Mugishima H (2006). Signal transduction via vascular endothelial growth factor (VEGF) receptors and their roles in atherogenesis. J. Atheroscler. Thromb. 13: 130-135.

Ohtani T, Mizuashi M, Ito Y and Aiba S (2007). Cadexomer as well as cadexomer iodine induces the production of proinflammatory cytokines and vascular endothelial growth factor by human macrophages. Exp. Dermatol. 16: 318323.

Pasmant E, de Beauvoir C, Plessier A, Labreuche J, et al. (2010). VKORC1 and CYP2C9 genetic polymorphisms in hepatic or portal vein thrombosis. Thromb. Res. 126: e134-e136.

Shibuya M (2006). Differential roles of vascular endothelial growth factor receptor-1 and receptor-2 in angiogenesis. $J$. Biochem. Mol. Biol. 39: 469-478.

Slusher KB (2010). Factor V Leiden: a case study and review. Dimens. Crit. Care Nurs. 29: 6-10.

Smalberg JH, Koehler E, Darwish MS, Plessier A, et al. (2011). The JAK2 46/1 haplotype in Budd-Chiari syndrome and 
portal vein thrombosis. Blood 117: 3968-3973.

Smira G, Gheorghe L, Iacob S, Coriu D, et al. (2010). Budd Chiari syndrome and V617F/JAK 2 mutation linked with the myeloproliferative disorders. J. Gastrointestin. Liver Dis. 19: 108-109.

Tondeur S, Boutruche S, Biron-Andréani C and Schved JF (2009). Prevalence of the JAK2 V617F mutation associated with splanchnic vein thrombosis. A 10-year retrospective study. Thromb. Haemost. 101: 787-789.

Wang XL, Xu LY, Zhang HT, Li JM, et al. (2007). Effects on endothelial cell proliferation induced by different concentration of iodine in vitro. J. Shandong Univ. (Health Sci.) 45: 310-312.

Wang Y, Zhang H, Guo CH and Fan QY (2008). Pathologic and etiologic studies of membrane obstruction of the inferior vena cava in Budd-Chiari syndrome. J. Interventional. Radiol. 17: 500-503.

Wlazłowski M, Kaczorowska-Hać B, Klukowska A and Balcerska A (2011). Budd-Chiari syndrome in 16 year old girl with essential thrombocytosis. Med. Wieku. Rozwoj. 15: 140-142.

Yadav D, Chandra J, Sharma S and Singh V (2012). Essential thrombocytosis and antiphospholipid antibody syndrome causing chronic Budd-Chiari syndrome. Indian J. Pediatr. 79: 538-540.

Yamana K, Bilim V, Hara N, Kasahara T, et al. (2005). Prognostic impact of FAS/CD95/APO-1 in urothelial cancers: decreased expression of Fas is associated with disease progression. Br. J. Cancer 93: 544-551. 